\title{
Review of Radiology: App for Android by the Radiological Society of North America (RSNA)
}

\author{
Andy Wai Kan Yeung ${ }^{1}$ (D) - W. Keung Leung ${ }^{1}$
}

Published online: 14 November 2016

(C) Society for Imaging Informatics in Medicine 2016

\section{App Specs}

App Icon URL: https://h6.ggpht.com/h7vLZmMU0E53067 F s 2 i C m D e T 3 K d F b k y 6 Z w I X P P R B e z 5 C W 5 aMKsuHVIIKDsRv2rTCHGRE=w300-rw

App Name: Radiology

App Developer: Radiological Society of North America (RSNA)

App Developer Website: http://www.rsna.org/

App Price: Free

Apple App Store URL: https://itunes.apple. com/us/app/radiology-radiological-society/id741007884 $? \mathrm{mt}=8$

Google Play Store URL: https://play.google. $\mathrm{com} /$ store/apps/details?id=com.sheridan.rsna.radiology

Category: Journal, educational

Tags: Free, Android and iOS compatible, radiology, journal Works Offline: $\mathrm{N}$

FDA Approval: NA

Promotion Code: NA

Andy Wai Kan Yeung

ndyeung@hku.hk

1 Faculty of Dentistry, The University of Hong Kong, Hong Kong, Hong Kong

\section{Quick Review}

(1 star: lowest $/ 5$ stars: highest)

Overall Rating (1-5): 4

Content (1-5): 4

Usability (1-5): 4

Pros: The app allows immediate access to the archive of Radiology journal published by Radiological Society of North America (RSNA). Loading time is quick.

Cons: The user interface does not allow customization.

At A Glance: It is a must-have app for healthcare workers who will stay updated by accessing Radiology journal.

\section{Full Review}

\section{Introduction}

Radiology is an app specially designed for accessing the Radiology journal published by RSNA. Therefore, the user should be able to browse through its content and conduct searches to identify specific articles via a few simple clicks. Both iOS and Android versions are available, and this article has reviewed its Android version.

\section{Purpose/Features/Content}

The purpose of the app is to provide convenient access to all articles published in Radiology journal. Once the user turns on the app, there is a bookshelf displaying the latest issues of the journal. A menu is readily available by swiping the left side of the screen. The top part of the menu contains buttons for various actions to retrieve bookmarked articles and access adjunctive materials provided by RSNA, such as YouTube videos, podcasts, and Facebook page of RSNA (Fig. 1). 


\section{Radiology}

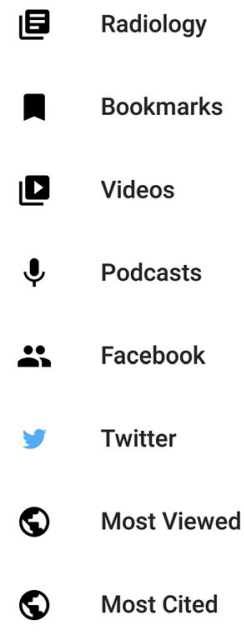

Fig. 1 Top part of the menu. It contains many links to adjunctive resources

After scrolling down the menu, a list of topics is displayed (Fig. 2). Articles are sorted according to their categories and

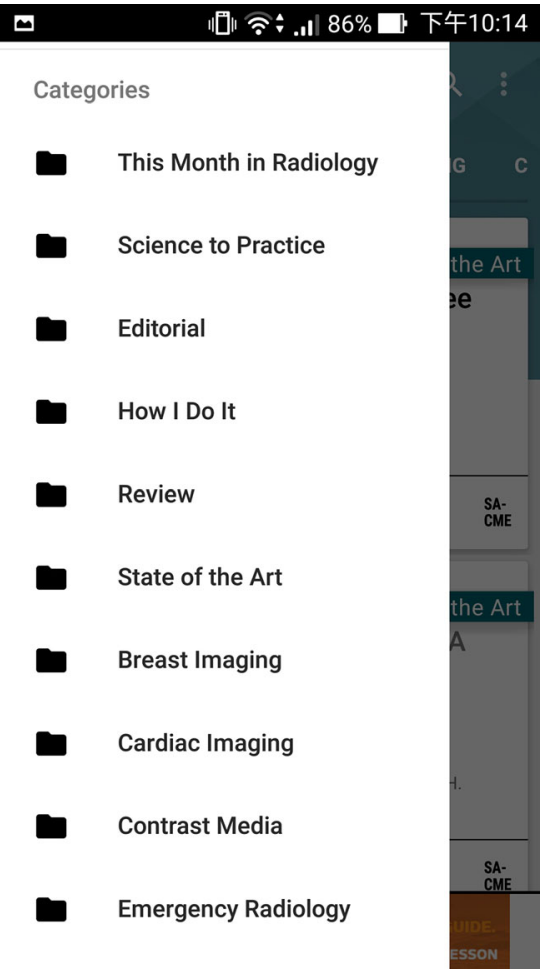

Fig. 2 Bottom part of the menu. Articles can be accessed according to categories this allows the user to concentrate on articles in specific topics of interest. Swiping the right side of the screen activates the search function. The user can find articles by typing in keywords, author, and/or title (Fig. 3).

\section{Usability}

The app serves the purposes efficiently. The font size and background color allows comfortable reading. The search is effective. Loading time is quick. Full texts and PDFs are available via institutional subscriptions.

\section{Good}

Both main text (Fig. 4) and figures (Fig. 5) are displayed clearly. The links to figures and references are shaded with a sharp contrast for easy clicking. Font size can be adjusted as small, medium, or large. Depending on the storage space of the user's device, the caching level can be adjusted as low, medium, or high. This controls the amount of data downloaded and stored into the user's device for faster loading time and article retrieval.

\section{Room for Improvement}

The swiping action to activate the search function may not work well in landscape mode. The user's fingers may accidentally touch the "back," "home," or "task switcher" buttons

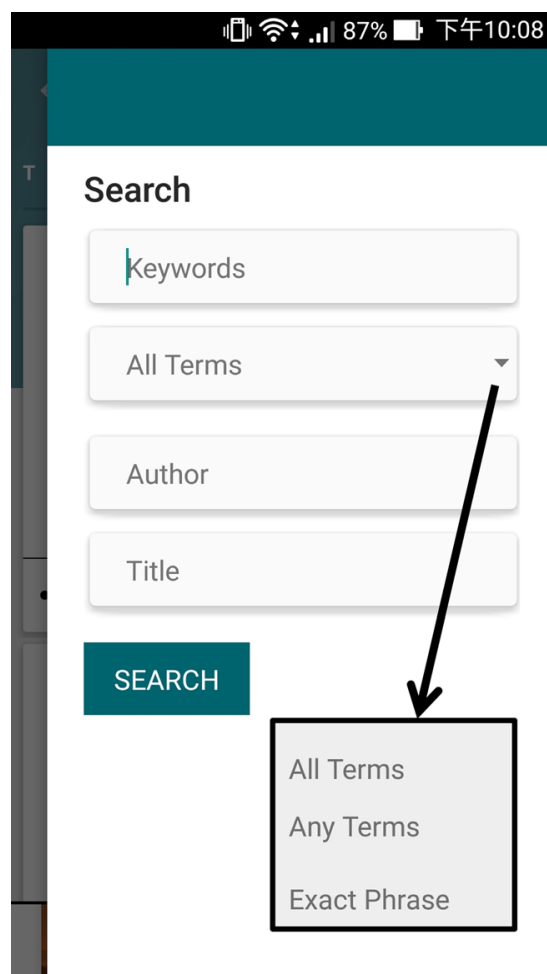

Fig. 3 Search function. Articles can be found by searching keywords, author, and/or title 
Ш

\section{$\equiv$ Soft-Tissu... $<>\quad C$ :}

histologically identical to adipose fat

(7) ). The incidence of lipomas is up to 2.1 per 100 individuals ( 7 ). Lipomas are radiolucent on radiographs and computed tomographic (CT) images and are isointense relative to subcutaneous fat on MR images obtained with all pulse sequences ( 7 ). The classic lipoma is composed entirely of fat, without areas of nodularity or thickened septations (8) ( Fig 1 ).

Of note, a substantial percentage of benign lipomas demonstrate nonadipose features. In a study by Kransdorf et al ( 8 ), 31\% (11 of 35) of lipomas showed nonadipose content, which the authors attributed to fat necrosis and

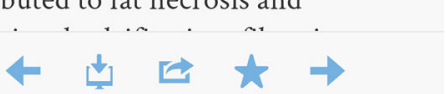

Fig. 4 Reading the main text. Font size and spacing allow comfortable reading

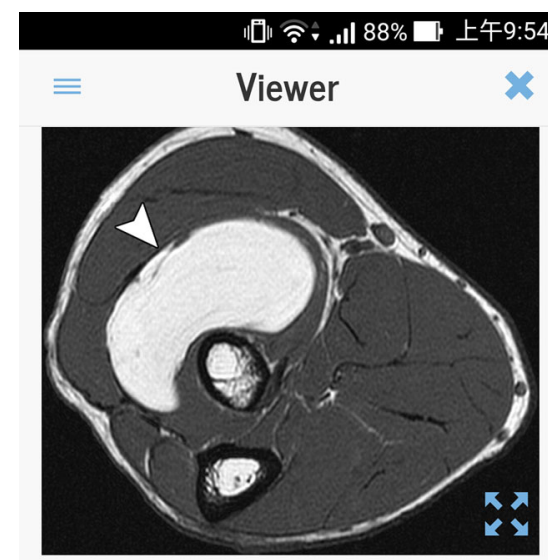

Figure la: Axial MR images in 49-yearold man show lipoma (arrowhead) in upper forearm. (a) Spin-echo (SE) T1weighted MR image shows uniformly hyperintense lesion with SI identical to that of subcutaneous fat. (b) Fatsuppressed spoiled gradient-recalled acquisition in the steady state (SPGR) T1weighted MR image shows uniform fat

Fig. 5 Viewing a figure. The figure can be magnified while maintaining reasonable resolution, and the figure legend is attached at the bottom

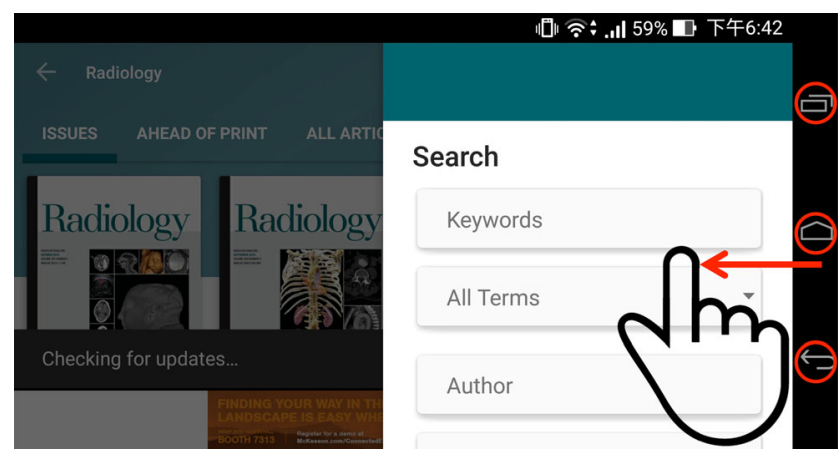

Fig. 6 Swiping the phone in landscape mode. The fingers may accidentally touch the buttons at the edge of the screen when the user tries to activate search function by swiping from the right edge of the screen

at the side of the screen instead of activating the search (Fig. 6). It would be great if the swiping action can be performed within the screen without the need to cross the screen edge. Also, it would be great if the menu can be customized. For example, if the user can replace the folder icons next to each topic with special ones (Fig. 2), the words may even be eliminated to allow a smaller yet more compact menu without the need to scroll down. 